Bull. Austral. Math. Soc.

$11 \mathrm{G} 05,14 \mathrm{H} 52$

VoL. $70(2004) \quad[125-142]$

\title{
TRACE OF FROBENIUS ENDOMORPHISM OF AN ELLIPTIC CURVE WITH COMPLEX MULTIPLICATION
}

\author{
NOBURO ISHII
}

Let $E$ be an elliptic curve with complex multiplication by $R$, where $R$ is an order of discriminant $D<-4$ of an imaginary quadratic field $K$. If a prime number $p$ is decomposed completely in the ring class field associated with $R$, then $E$ has good reduction at a prime ideal $\mathfrak{p}$ of $K$ dividing $p$ and there exist positive integers $u$ and $v$ such that $4 p=u^{2}-D v^{2}$. It is well known that the absolute value of the trace $a_{\mathfrak{p}}$ of the Frobenius endomorphism of the reduction of $E$ modulo $\mathfrak{p}$ is equal to $u$. We determine whether $a_{\mathfrak{p}}=u$ or $a_{\mathrm{p}}=-u$ in the case where the class number of $R$ is 2 or 3 and $D$ is divisible by 3,4 or 5 .

\section{INTRODUCTION}

Let $K=\mathbb{Q}(\sqrt{-m})$ be an imaginary quadratic field, where $m$ is a square-free positive integer. Let $R$ be an order of $K$ of conductor $f_{0}$ with a basis $\{1, \omega\}$ over $\mathbb{Z}$. We denote by $d(R)$ and $h(R)$ the discriminant and the class number of $R$ respectively. Let $f$ be the smallest positive integer such that $f \sqrt{-m} \in R$. Then we have $f=f_{0} / 2$ if $m \equiv 3 \bmod 4$ and $f_{0}$ is even, otherwise $f=f_{0}$. Let $E$ be an elliptic curve with complex multiplication by $R$ and denote by $j(E)$ the $j$-invariant of $E$. We may assume that $E$ is defined by a short Weierstrass equation: $y^{2}=x^{3}+A x+B, A, B \in F=\mathbb{Q}(j(E))$. First, we introduce the notation used in the following. For an endomorphism $\lambda$ of $E$, the kernel of $\lambda$ is denoted by $E[\lambda]$. For a prime ideal $\mathfrak{p}$ of $F$, we denote by $\ell_{\mathfrak{p}}$ the relative degree of $\mathfrak{p}$ over $\mathbb{Q}$. If $E$ has good reduction at $\mathfrak{p}$, then we denote by $\tilde{E}_{\mathfrak{p}}$ the reduction of $E$ modulo $\mathfrak{p}$. For a point $P$ of $E$ we denote by $P^{\sim}$ the reduction of $P$ modulo $\mathfrak{p}$. Further we denote by $\varphi_{\mathfrak{p}}$ the Frobenius endomorphism of $\tilde{E}_{\mathfrak{p}}$ and by $a_{\mathfrak{p}}(E)$ the trace of $\varphi_{\mathfrak{p}}$. By $\mathbb{F}_{q}$, we denote the finite field of $q$-elements. If $\tilde{E}_{\mathfrak{p}}$ is defined over $\mathbb{F}_{q}$, then $\tilde{E}_{\mathfrak{p}}\left(\mathbb{F}_{q}\right)$ denotes the group of $\mathbb{F}_{q}$-rational points of $\tilde{E}_{\mathfrak{p}}$.

Now let $p$ be an odd prime number and $\mathfrak{p}$ a prime ideal of $F$ dividing $p$. Let us assume that $p$ and $\mathfrak{p}$ satisfy the following condition:

Received 9th February, 2004

Partially supported by Grand-in-Aid for Scientific Research No.12640036 and No.15540042. The author would like to express his hearty gratitude to Professor M. Kaneko for offering the table of class equations and to Tomoko Ibata for writing and running the computer program required for this work.

Copyright Clearance Centre, Inc. Serial-fee code: 0004-9727/04 \$A2.00+0.00. 
(1) $p$ splits completely in $K, p$ is prime to $f$, and $E$ has good reduction at $\mathfrak{p}$. Then by complex multiplication theory (see [11, Chapter III]), we know that $E$ has ordinary good reduction at $\mathfrak{p}$ and the endomorphism ring of $\tilde{E}_{\mathfrak{p}}$ is isomorphic to $R$ (see [6, Theorem 12, 13.4]). Further $K(j(E))$ is the ring class field of $K$ of conductor $f_{0}$ (see [3, Section 9]). Since $\mathfrak{p}$ is of relative degree $\ell_{\mathfrak{p}}$, there exist positive integers $u_{p}$ and $v_{p}$ such that

$$
4 p^{\ell_{p}}=u_{p}^{2}+m f^{2} v_{p}^{2},\left(u_{p}+v_{p} f \sqrt{-m}\right) / 2 \in R,\left(u_{p}, p\right)=1 .
$$

By the assumption, we may write $\varphi_{\mathfrak{p}}=\left(a_{\mathfrak{p}}(E)+b_{\mathfrak{p}}(E) f \sqrt{-m}\right) / 2=\alpha+\beta \omega$, where $b_{p}(E), \alpha$ and $\beta$ are integers. It is known that the group $\tilde{E}_{\mathfrak{p}}\left(\mathbb{F}_{p_{\mathfrak{p}}}\right)$ is of order $N_{p}(E)$ $=p^{\ell_{\mathfrak{p}}}+1-a_{\mathfrak{p}}(E)$ and is isomorphic to the group $\mathbb{Z} /\left(N_{\mathfrak{p}}(E) / d\right) \mathbb{Z} \oplus \mathbb{Z} / d \mathbb{Z}$, where $d$ is the greatest common divisor of $\alpha-1$ and $\beta$. On the other hand, if $d(R)<-4$, then we have easily $a_{\mathfrak{p}}(E)=\varepsilon_{\mathfrak{p}} u_{p}$, where $\varepsilon_{\mathfrak{p}}=1$ or -1 . It is easy to find $u_{p}$ for a given number $4 p^{\ell_{p}}$ such that $4 p^{\ell_{p}}=u_{p}^{2}+m f^{2} v_{p}^{2},\left(u_{p}, p\right)=1$. Therefore if we determine $\varepsilon_{p}$, then we can compute the numbers $N_{\mathfrak{p}}(E)$ and $d$ quickly. The problem of determining $\varepsilon_{\mathfrak{p}}$ in the case $h(R)=1$ has been solved by Rajwade, Joux and Morain and others. See [5] for the references to their results. In the case $h(R)=2$, this problem is solved for only one case of the order of discriminant -20 , by Leprévost and Morain ([7]), using the results of $[1,2]$ for the character sum of Dickson polynomial of degree 5 .

The purpose of this article is to determine $\varepsilon_{\mathfrak{p}}$ for an elliptic curve $E$ having complex multiplication by $R$ and for prime ideals $\mathfrak{p}$ of $F$ satisfying (1), where $R$ is an order such that $h(R)=2$ or 3 and $m f^{2}$ is divided by 3,4 or 5 . Thus $R$ are orders of discriminant

$$
\begin{aligned}
d(R)=-15,-20, & -24,-32,-35,-36,-40,-48,-51,-60,-64,-72 \\
& -75,-99,-100,-108,-112,-115,-123,-147,-235,-243,-267 .
\end{aligned}
$$

Further we assume that $j(E)$ is real to avoid tedious computation.

Our idea to solve the problem is as follows (for details see Section 2). Let $s$ be a divisor of $f^{2} m$ and assume $s \geqslant 3$. We find a $F$-rational cyclic subgroup $C_{s}$ of $E[f \sqrt{-m}]$ of order $s$ and take a generator $Q$ of $C_{s}$. Consider the Frobenius isomorphism of $\sigma_{\mathfrak{p}}$ of $\mathfrak{p}$. Then $F$-rationality of $C_{s}$ shows $Q^{\sigma_{\mathfrak{p}}}=\left[r_{\mathfrak{p}}\right](Q)$ for an integer $r_{\mathfrak{p}}$. Using $Q^{\sim} \in \tilde{E}_{\mathfrak{p}}[f \sqrt{-m}]$ and $\left(Q^{\sigma_{\mathfrak{p}}}\right) \sim=\varphi_{\mathfrak{p}}\left(Q^{\sim}\right)$, we have

$$
\begin{aligned}
{[2]\left(\left[r_{\mathfrak{p}}\right](Q)\right)^{\sim} } & =[2]\left(Q^{\sigma_{\mathfrak{p}}}\right)^{\sim}=[2] \varphi_{\mathfrak{p}}\left(Q^{\sim}\right) \\
& =\left[\left(a_{\mathfrak{p}}(E)+b_{\mathfrak{p}}(E) f \sqrt{-m}\right)\right]\left(Q^{\sim}\right)=\left[a_{\mathfrak{p}}(E)\right]\left(Q^{\sim}\right) .
\end{aligned}
$$

This shows that $a_{\mathfrak{p}}(E) \equiv 2 r_{\mathfrak{p}} \bmod s$. Therefore the number $\varepsilon_{\mathfrak{p}}$ is determined by the condition $\varepsilon_{\mathrm{p}} u_{\mathrm{p}} \equiv 2 r_{\mathrm{p}} \bmod s$. This argument reduces our original problem to a problem of finding a point $Q$ and of determining $r_{\mathfrak{p}}$ for a given prime ideal $\mathfrak{p}$. In Section 2, we give auxiliary results to find the cyclic subgroup $C_{s}$ and a generator $Q$. If $s$ is an odd prime number, then we show, in Proposition 2.8 of Section 2, that the s-division polynomial 
$\Psi_{s}(x, E)$ of $E$ has a unique $F$-rational factor $H_{1, E}(x)$ of degree $(s-1) / 2$ and that the point $Q$ is obtainable from a solution of $H_{1, E}(x)=0$. In Section 3 we determine $r_{p}$ in the case $f^{2} m$ is divided by 3 or 4 and in Section 4 in the case $f^{2} m$ is divided by 5 . Though we deal with a specified elliptic curve $E$ for each order $R$, a similar result is easily obtained for any elliptic curve $E^{\prime}$ of the $j$-invariant $j(E)$, because $E^{\prime}$ is a quadratic twist of $E$ and $a_{\mathfrak{p}}\left(E^{\prime}\right)$ is the product of $a_{\mathfrak{p}}(E)$ and the value at $\mathfrak{p}$ of the character associated with the twist.

In the following, we assume any elliptic curve is defined by a short Weierstrass equation.

\section{ThE SUBgRoups of $E[f \sqrt{-m}]$ AND DECOMPOSITION OF DIVISION POLYNOMIALS}

2.1. Let $E$ be an elliptic curve with complex multiplication by $R$. By the definition of $f$, we have $f \sqrt{-m} \in R$.

Proposition 2.1. The group $E[f \sqrt{-m}]$ is cyclic of order $f^{2} m$.

Proof: By [8, Proposition 2.1], we know $E[f \sqrt{-m}]$ is isomorphic to $R / f \sqrt{-m} R$. Let $f$ be odd and $m \equiv 3 \bmod 4$. Then $R=\mathbb{Z} \oplus f \omega \mathbb{Z}$, where $\omega=(1+\sqrt{-m}) / 2$. Further $f \sqrt{-m} R=f(2 \omega-1) \mathbb{Z} \oplus f^{2}(\omega-(m+1) / 2) \mathbb{Z}$. Put $\xi=f \omega-f(m f+1) / 2 \in f \sqrt{-m} R$. Then we have $f(2 \omega-1)=2 \xi+m f^{2}, f^{2}(\omega-(m+1) / 2)=f \xi+(f-1) f^{2} m / 2$. This shows that $\left\{f^{2} m, \xi\right\}$ is a basis of $f \sqrt{-m} R$ over $\mathbb{Z}$. Since $\{1, \xi\}$ is a basis of $R$ over $\mathbb{Z}$, $R / f \sqrt{-m} R$ is a cyclic group of order $f^{2} m$. The assertion for the other case is easily obtained.

LEMma 2.2. Let $r$ be a fixed prime number. Then there exist infinitely many prime numbers of the form $u^{2}+v^{2} f^{2} m$, where $u$ and $v$ are integers and $v$ is prime to $r$.

Proof: Consider the ideal groups $G_{0}$ and $P_{0}$ of $K$ such that

$$
G_{0}=\{\mathfrak{a} \mid \mathfrak{a} \text { is prime to } 2 r f m\}, P_{0}=\{(\alpha) \mid \alpha \equiv 1 \bmod 2 r f \sqrt{-m}\} .
$$

Then $P_{0}$ is a subgroup of $G_{0}$ of finite index and by Tshebotareff's density theorem, in each factor class there exist infinitely many prime ideals of degree 1 . Let $\gamma=u_{0}+v_{0} f \sqrt{-m}$ such that ideal $(\gamma) \in G_{0}$ and $u_{0}, v_{0} \in \mathbb{Z}$ and further $v_{0}$ is prime to $r$. Then every integral ideal of the class $(\gamma) P_{0}$ has a generator of the form $u_{1}+v_{1} f \sqrt{-m}\left(u_{1}, v_{1} \in \mathbb{Z}, r \nmid v_{1}\right)$. Thus we have our assertion.

In the following, let $p$ be an odd prime number and $\mathfrak{p}$ a prime ideal of $F$ dividing $p$ and assume that $p$ and $\mathfrak{p}$ satisfy the condition (1).

LemMa 2.3. Let $s$ be an odd prime number dividing $f^{2} m$. Let $q=p^{\ell_{p}}$. Assume that $q=u^{2}+v^{2} f^{2} m,(v, p s)=1$ or $4 q=u^{2}+v^{2} f^{2} m,(v, 2 s p)=1$. Then we have

$$
\tilde{E}_{\mathrm{p}}[s] \cap \tilde{E}_{\mathfrak{p}}[f \sqrt{-m}] \backslash\{0\}=\left\{P=(\alpha, \beta) \in \tilde{E}_{\mathfrak{p}}[s] \mid s \nmid\left[\mathbb{F}_{q}(\alpha): \mathbb{F}_{q}\right]\right\},
$$

where $\left[\mathbb{F}_{q}(\alpha): \mathbb{F}_{q}\right]$ denotes the degree of the field $\mathbb{F}_{q}(\alpha)$ over $\mathbb{F}_{q}$. 
Proof: By the assumption, $\tilde{E}_{\mathrm{p}}$ is defined over $\mathbb{F}_{q}$. First we assume the Frobenius endomorphism $\varphi_{\mathfrak{p}}$ is given by $\varphi_{\mathfrak{p}}=(u+v f \sqrt{-m}) / 2$, if necessary, after replacing $u$ by $-u$ or $v$ by $-v$. Let $P=(\alpha, \beta) \in \tilde{E}_{\mathfrak{p}}[s]$. If $P \in \tilde{E}_{\mathfrak{p}}[f \sqrt{-m}]$, then, for $h=(s-1) / 2$, we have $\varphi_{\mathfrak{p}}^{h}\left(\left[2^{h}\right](P)\right)=\left[u^{h}\right](P)$. Since $2^{h}, u^{h} \equiv \pm 1 \bmod s$, we have $\varphi_{\mathfrak{p}}^{h}(P)= \pm P$. This shows $\left[\mathbb{F}_{q}(\alpha): \mathbb{F}_{q}\right] \leqslant(s-1) / 2$. Conversely let $P=(\alpha, \beta) \in \tilde{E}_{\mathfrak{p}}[s], s \nmid k=\left[\mathbb{F}_{q}(\alpha): \mathbb{F}_{q}\right]$ and $r=q^{k}$. Since $\varphi_{\mathfrak{p}}^{k}(P)=\left(\alpha^{r}, \beta^{r}\right)=\left(\alpha, \beta^{r}\right)=\varepsilon P(\varepsilon= \pm 1)$, we have $[(u+v f \sqrt{-m}) / 2)^{k}$ $-\varepsilon](P)=0$. Since $P \in \tilde{E}_{\mathfrak{p}}[s]$ and $s \mid f^{2} m$, we have $\left[\left(u^{k}-2^{k} \varepsilon\right)+k u^{k-1} v f \sqrt{-m}\right](P)=0$ and $\left[\left(u^{k}-2^{k} \varepsilon\right)^{2}+\left(k u^{k-1} v\right)^{2} f^{2} m\right](P)=0$. Thus $\left[\left(u^{k}-2^{k} \varepsilon\right)^{2}\right](P)=0$. Since the order of $P$ is $s$, we see $\left[\left(u^{k}-2^{k} \varepsilon\right)\right](P)=0$ and $\left[k u^{k-1} v f \sqrt{-m}(P)=0\right.$. By the assumption, $k, u$ and $v$ are prime to $s$. Therefore we conclude $[f \sqrt{-m}](P)=0$. Hence $P \in \tilde{E}_{\mathfrak{p}}[f \sqrt{-m}]$. In the case $\varphi_{\mathfrak{p}}=u+v f \sqrt{-m}$, the same argument holds true.

Corollary 2.4. Let $\Psi_{s}\left(x, \tilde{E_{p}}\right)$ be the s-division polynomial of $\tilde{E}_{\mathrm{p}}$. Then $\Psi_{s}\left(x, \tilde{E}_{\mathfrak{p}}\right)$ is the product of two $\mathbb{F}_{q}$-rational polynomials $h_{1}(x)$ and $h_{2}(x)$ such that $h_{1}(x)$ is of degree $(s-1) / 2$ and the degree of every irreducible factor of $h_{2}(x)$ is divided by $s$. Further the solutions of $h_{1}(x)=0$ consist of all distinct $x$-coordinates of non-zero points in $\tilde{E}_{\mathrm{p}}[s] \cap \tilde{E}_{\mathrm{p}}[f \sqrt{-m}]$.

Proof: Since $p$ is prime to $f^{2} m$, by Proposition $2.1, \tilde{E}_{\mathrm{p}}[s] \cap \tilde{E}_{\mathrm{p}}[f \sqrt{-m}]$ is a $\mathbb{F}_{q}$-rational cyclic group of order $s$. Thus if we put $h_{1}(x)=\prod_{\alpha}(x-\alpha)$, where $\alpha$ runs over all distinct $x$-coordinates of non-zero points in $\tilde{E}_{\mathfrak{p}}[s] \cap \tilde{E}_{\mathfrak{p}}^{\alpha}[f \sqrt{-m}]$, then $h_{1}(x)$ is $\mathbb{F}_{q}$-rational and of degree $(s-1) / 2$. The assertion for $h_{2}(x)$ follows immediately from Lemma 2.3.

LEMMA 2.5. Let $4 \mid f^{2} m$ and $q=p^{\ell_{p}}=u^{2}+v^{2} f^{2} m,(v, 2)=1$. Let $Q_{1}$ be a point of order 4 of $\tilde{E}_{\mathfrak{p}}[f \sqrt{-m}]$ and $Q_{2}$ a point of $\tilde{E}_{\mathfrak{p}}$ such that $[2]\left(Q_{1}\right)=[2]\left(Q_{2}\right)$ and $Q_{2} \neq \pm Q_{1}$. Then the $x$-coordinates $x_{1}$ and $x_{2}$ of $Q_{1}$ and $Q_{2}$ are all $\mathbb{F}_{q}$-rational solutions of $\Psi_{4}\left(x, \tilde{E}_{\mathfrak{p}}\right) / y=0$. Furthermore let $y^{2}=h(x)$ be the equation of $\tilde{E}_{\mathfrak{p}}$. Assume that $\varphi_{\mathfrak{p}}=u+v f \sqrt{-m}$. Then, of two elements $x_{1}$ and $x_{2}$, only $x_{1}$ satisfies the relation $\left(h\left(x_{1}\right) / \mathfrak{p}\right)=(-1)^{(u-1) / 2}$, where $(/ \mathfrak{p})$ denotes the Legendre symbol for $\mathfrak{p}$.

Proof: Since $\tilde{E}_{\mathrm{p}}[f \sqrt{-m}]$ is a $\mathbb{F}_{q}$-rational cyclic group, we see $x_{1}$ and $x_{2}$ are $\mathbb{F}_{q}$ -rational. Let $\alpha$ be a $\mathbb{F}_{q}$-rational root of $\Psi_{4}\left(x, \tilde{E}_{\mathrm{p}}\right) / y=0$ and put $S=(\alpha, \beta)$. Then $S$ is a 4-division point of $\tilde{E}_{\mathrm{p}}$ and we have

$$
\varphi_{p}(S)=[u+v f \sqrt{-m}](S)=\left(\alpha^{q}, \beta^{q}\right)=(\alpha, \pm \beta)=[\varepsilon](S),(\varepsilon= \pm 1)
$$

Thus we have $[(u-\varepsilon)+v f \sqrt{-m}](S)=0$. This shows $\left[(u-\varepsilon)^{2}+v^{2} f^{2} m\right](S)=0$. Since the order of $S$ is $4, u-\varepsilon$ is divided by 2 . Thus $[f \sqrt{-m}]([2] S)=0$. Since $[2]\left(Q_{1}\right)$ is the only one point of degree 2 in $\tilde{E}_{\mathrm{p}}[f \sqrt{-m}]$, we have $[2](S)=[2]\left(Q_{1}\right)$. This shows that $S$ 
equals to one of $\pm Q_{1}$ and $\pm Q_{2}$. Therefore $\alpha$ equals to $x_{1}$ or $x_{2}$. Let $P=(x, y)$ be a point of $\tilde{E}_{\mathrm{p}}$ of order 4 such that $x \in \mathbb{F}_{q}$. Then

$$
\begin{aligned}
\varphi_{\mathfrak{p}}(P) & =\left(x^{q}, y^{q}\right)=\left(x, y h(x)^{(q-1) / 2}\right) \\
& =[(h(x) / \mathfrak{p})](P)=[u](P)+[v f \sqrt{-m}](P) .
\end{aligned}
$$

Therefore we have $(h(x) / \mathfrak{p}) \equiv u \bmod 4$ if and only if $P \in \tilde{E}_{\mathfrak{p}}[f \sqrt{-m}]$.

2.2. Let $s$ be a positive divisor of $f^{2} m$ and $s \geqslant 3$. By Proposition 2.1, there exists a unique subgroup $C_{s}$ of $E[f \sqrt{-m}]$ of order $s$. Let $Q=\left(x_{Q}, y_{Q}\right)$ be a generator of $C_{s}$. Consider the fields $L=F\left(x_{Q}\right)$ and $M=F(Q)$. Since $E[f \sqrt{-m}]$ is $F$-rational, $C_{s}$ is $F$-rational and the field $M$ is an Abelian extension over $F$. By class field theory, the Galois group $G$ of $M$ over $F$ is isomorphic to an ideal class group $\mathfrak{G}$ of $F$. For an ideal class $\mathfrak{C} \in \mathfrak{G}$, let $\sigma_{\mathfrak{C}}$ be the isomorphism of $G$ corresponding to $\mathfrak{C}$. Then we have

THEOREM 2.6. Let $\mathfrak{C}$ be the class represented by $\mathfrak{p}$ and $Q^{\sigma_{\mathfrak{C}}}=\left[r_{\mathfrak{C}}\right](Q)$. Then we have $a_{\mathfrak{p}}(E) \equiv 2 r_{\mathfrak{C}} \bmod s$. Further if $a_{\mathfrak{p}}(E)$ is even, then we have $a_{\mathfrak{p}}(E) / 2 \equiv r_{\mathfrak{C}} \bmod s$.

Proof: Let $\varphi_{\mathfrak{p}}=\left(a_{\mathfrak{p}}(E)+b_{\mathfrak{p}}(E) f \sqrt{-m}\right) / 2$. Since $\left(Q^{\sigma_{\mathfrak{C}}}\right)^{\sim}=\varphi_{\mathfrak{p}}\left(Q^{\sim}\right)$, we see

$$
\begin{aligned}
{[2]\left(\left[r_{\mathfrak{c}}\right](Q)\right)^{\sim} } & =[2]\left(Q^{\sigma_{\mathfrak{c}}}\right)^{\sim}=[2] \varphi_{\mathfrak{p}}\left(Q^{\sim}\right) \\
& =\left[\left(a_{\mathfrak{p}}(E)+b_{\mathfrak{p}}(E) f \sqrt{-m}\right)\right]\left(Q^{\sim}\right)=\left[a_{\mathfrak{p}}(E)\right]\left(Q^{\sim}\right) .
\end{aligned}
$$

Since $p$ is prime to $s, Q^{\sim}$ is of order $s$. Thus $a_{\mathfrak{p}}(E) \equiv 2 r_{\mathfrak{C}} \bmod s$. If $a_{\mathfrak{p}}(E)$ is even, then $\varphi_{\mathfrak{p}}=\left(a_{\mathfrak{p}}(E) / 2\right)+\left(b_{\mathfrak{p}}(E) / 2\right) f \sqrt{-m}$. By a similar argument we have $\left[a_{\mathfrak{p}}(E) / 2\right]\left(Q^{\sim}\right)$ $=\left[r_{\mathfrak{c}}\right]\left(Q^{\sim}\right)$. This shows the remaining assertion.

PROPOSITION 2.7. Let $s$ be an odd prime divisor of $f^{2} m$. If $p^{\ell_{p}} \equiv 1 \bmod s$, then

$$
a_{\mathfrak{p}}(E) \equiv 2\left(\frac{y_{Q}^{2}}{\mathfrak{p}}\right) \bmod s
$$

Proof: Since we have $4 p^{\ell_{\mathfrak{p}}}=a_{\mathfrak{p}}(E)^{2}+b_{\mathfrak{p}}(E)^{2} f^{2} m$, Theorem 2.6 shows that $r_{\mathfrak{c}} \equiv \pm 1$ $\bmod s$. Thus $x_{Q} \in \mathbb{F}_{q}$. By the similar argument in the last part of Lemma 2.5, we have our assertion.

PROPOSITION 2.8. Let $s$ be an odd prime divisor of $f^{2} m$ and $\Psi_{s}(x, E)$ the $s$-division polynomial of $E$. Then $\Psi_{s}(x, E)$ is the product of two $F$-rational polynomials $H_{1, E}(x)$ and $H_{2, E}(x)$ such that $H_{1, E}(x)$ is of degree $(s-1) / 2$ and every irreducible factor of $H_{2, E}(x)$ is of degree $s$. Further the solutions of $H_{1, E}(x)=0$ consist of all distinct $x$-coordinates of non-zero points of $C_{s}$.

Proof: Let $H_{1, E}(x)=\prod_{t}(x-t)$, where $t$ runs over all distinct $x$-coordinates of non-zero points of $C_{s}$. Since $C_{s}$ is $F$-rational, we see $H_{1, E}(x)$ is $F$-rational of degree $(s-1) / 2$ and clearly it divides $\Psi_{s}(x, E)$. By Lemma 2.2, we can choose an odd 
prime $p$ and a prime ideal $\mathfrak{p}$ dividing $p$ such that they satisfy (1) and $p$ is of the form $p=u^{2}+v^{2} f^{2} m,(v, s)=1$, and further the reduction of $\Psi_{s}(x, E)$ modulo $\mathfrak{p}$ is equal to $\Psi_{s}\left(x, \tilde{E}_{\mathfrak{p}}\right)$. Take a point $P \in E[s] \backslash C_{s}$ and put $Q=[f \sqrt{-m}](P)$. Clearly, we have $Q \in E[f \sqrt{-m}]$ and $E[s]=\langle P\rangle \oplus\langle Q\rangle$. Let $G_{1}$ be the Galois group of $F(E[s])$ over $F$. By the representation of $G_{1}$ on $E[s]$ with the basis $\{P, Q\}, G_{1}$ is identified with a subgroup of the group

$$
G_{0}=\left\{\left(\begin{array}{cc}
a & 0 \\
b & \pm a
\end{array}\right) \mid a \in \mathbb{F}_{s}^{\times}, b \in \mathbb{F}_{s}\right\} .
$$

Consider the subgroups of $G_{0}$ :

$$
H=\left\{\left(\begin{array}{cc}
a & 0 \\
0 & \pm a
\end{array}\right) \mid a \in \mathbb{F}_{s}^{\times}\right\}, N=\left\{\left(\begin{array}{ll}
1 & 0 \\
b & 1
\end{array}\right) \mid b \in \mathbb{F}_{s}\right\} .
$$

Then we see $G_{0}=H N, G_{0} \triangleright N$ and $H \cap N=\left\{1_{2}\right\}$, where $1_{2}$ is the unit matrix. Since the order of $N$ is $s$ and $s$ is prime, we know that $G_{1} \supset N$ or $G_{1} \cap N=\left\{1_{2}\right\}$. Let $\Omega$ be the set of all subgroups of order $s$ of $E[s]$. Then $\Omega$ consists of $s+1$ elements and $G_{1}$ operates on $\Omega$. By Corollary 2.4, the degree of every irreducible factor of $H_{2, E}(x)=\Psi_{s}(x, E) / H_{1, E}(x)$ is divided by $s$. Thus we know $C_{s}$ is one and the only one fixed point of $G_{1}$. First let us consider the case $G_{1} \supset N$. Then we have $G_{1}=H_{1} N, H_{1}=H \cap G_{1}$. Since $H_{1}$ is the fixed subgroup of $\langle P\rangle$, the orbit of $\langle P\rangle$ consists of $s$ elements. Therefore $\Omega$ decomposes into two orbits. In particular, for each $n, 1 \leqslant n \leqslant(s-1) / 2$, the $x$-coordinate of $[n] P$ has $s$ conjugates over $F$. Thus every irreducible factor of $H_{2, E}(x)$ is of degree $s$. Next consider the case $G_{1} \cap N=\left\{1_{2}\right\}$. Then the order of $G_{1}$ is a divisor of $2(s-1)$ and is prime to $s$. Since the order of a matrix $\left(\begin{array}{ll}a & 0 \\ b & a\end{array}\right),(b \neq 0)$ is divided by $s, G_{1} \operatorname{dose}$ not contain the matrices of this form. Therefore there exists an element $\lambda \in \mathbb{F}_{s}$ such that $G_{1}$ is contained in the subgroup

$$
\left\langle\alpha \cdot 1_{2},\left(\begin{array}{cc}
1 & 0 \\
\lambda & -1
\end{array}\right) \mid \alpha \in \mathbb{F}_{s}^{\times}\right\rangle
$$

This shows $\langle P+(\lambda / 2) Q\rangle$ is a fixed point. Thus we have a contradiction.

PROPOSITION 2.9. Let $4 \mid f^{2} m$. If $Q$ is a point of order 4 in $E[f \sqrt{-m}]$ and $T$ is a point of $E$ such that $[2](Q)=[2](T)$ and $T \neq \pm Q$, then the $x$-coordinates $x_{Q}$ and $x_{T}$ of $Q$ and $T$ are all $F$-rational solutions of $\Psi_{4}(x, E) / y=0$.

PROOF: Using Lemma 2.5 instead of Lemma 2.3 and tracing the argument in the first part of Proposition 2.8, we have the assertion.

In Section 4, to study the ideal class groups of $F$ corresponding to the Abelian extensions $L$ and $M$, we must determine conductors $f_{L}$ and $\mathfrak{f}_{M}$ of $L$ and $M$ over $F$. In next lemma, we shall give some results for the conductors. For a prime ideal $q$ and an 
integral ideal $\mathfrak{a}$ of $F$, we denote by $e_{\mathfrak{q}}(\mathfrak{a})$ the maximal integer $m$ such that $m \geqslant 0$ and $\mathfrak{q}^{m}$ dividing a.

Lemma 2.10. Let $Q$ be a point of $E$ of order $s$. Assume that $s$ is an odd prime number, $s>3$ and $Q$ generates a $F$-rational subgroup $\langle Q\rangle$. Let $L, M, \mathfrak{f}_{L}$ and $\mathfrak{f}_{M}$ be defined for $Q$ as above. If $\mathfrak{q}$ is a prime ideal of $F$ prime to $(2 s)$, then $e_{\mathfrak{q}}\left(f_{L}\right) \leqslant e_{\mathfrak{q}}\left(f_{M}\right)$ and $e_{\mathfrak{q}}\left(\mathfrak{f}_{M}\right)>0$ implies $e_{\mathfrak{q}}\left(\mathfrak{f}_{L}\right)>0$. Further if $E$ has good reduction at $\mathfrak{q}$, then $e_{\mathfrak{q}}\left(\mathfrak{f}_{M}\right)=0$.

Proof: Since $L$ is a subfield of $M$, clearly $e_{\mathfrak{q}}\left(\mathfrak{f}_{L}\right) \leqslant e_{\mathfrak{q}}\left(\mathfrak{f}_{M}\right)$. If $E$ has good reduction at $\mathfrak{q}$, then Néron-Ogg-Shafarevich criterion ([10, Proposition 4.1, Chapter VII]) shows that $e_{\mathfrak{q}}\left(\mathfrak{f}_{M}\right)=0$. We shall prove $e_{\mathfrak{q}}\left(\mathfrak{f}_{M}\right)>0$ implies $e_{\mathfrak{q}}\left(\mathfrak{f}_{L}\right)>0$. Assume that $\mathfrak{q}$ is ramified in $M$ and is unramified in $L$. Let $\mathfrak{Q}$ be a prime ideal of $M$ dividing $\mathfrak{q}$ and $M_{\mathfrak{Q}}$ the completion of $M$ with respect to $\mathfrak{Q}$. Further we denote by $k_{M}$ the residue field of $\mathfrak{Q}$. Let $E_{0}, E_{1}$ and $\tilde{E}_{n s}$ be the groups defined in [10, Chapter VII]. Since $E$ has additive reduction at $\mathfrak{q}$, by $\left[10\right.$, Theorem 6.1 , Chapter VII] we have $\left[E\left(M_{\mathfrak{Q}}\right): E_{0}\left(M_{\mathfrak{Q}}\right)\right]=w \leqslant 4$. Since $Q$ has order $s$, by replacing $Q$ by $[w] Q$ if necessary, we can assume that $Q \in E_{0}\left(M_{\mathfrak{Q}}\right)$. Let $\sigma$ be a non trivial element of inertia group of $\mathfrak{Q}$. Then since $x_{Q}^{\sigma}=x_{Q}$, we have $Q^{\sigma}=-Q$. By considering the reduction modulo $\mathfrak{q}$, we have $Q^{\sim}=-Q^{\sim}$. Therefore $Q^{\sim} \in \tilde{E}_{n s}\left(k_{M}\right)$ and $[2]\left(Q^{\sim}\right)=0$. Since the characteristic of $k_{M}$ is prime to 2, by [10, Propsositions 2.1 and 5.1, Chapter VII ], we know $Q^{\sim}=0$, thus, we have $Q \in E_{1}\left(M_{\mathfrak{Q}}\right)$. Consequently, by [10, Proposition 3.1, Chapter VII], we have $Q=0$. This contradicts that $Q \neq 0$.

Finally for $s=3,4,5$, we list $s$-division polynomials $\Psi_{s}(x, E)$ :

$$
\left\{\begin{array}{c}
\Psi_{3}(x, E)=3 x^{4}+6 A x^{2}+12 B x-A^{2} \\
\frac{\Psi_{4}(x, E)}{4 y}=x^{6}+5 A x^{4}+20 B x^{3}-5 A^{2} x^{2}-4 A B x-8 B^{2}-A^{3} \\
\Psi_{5}(x, E)=5 x^{12}+62 A x^{10}+380 B x^{9}-105 A^{2} x^{8}+240 A B x^{7} \\
-\left(300 A^{3}+240 B^{2}\right) x^{6}-696 A^{2} B x^{5}-\left(125 A^{4}+1920 A B^{2}\right) x^{4} \\
-\left(1600 B^{3}+80 B A^{3}\right) x^{3}-\left(50 A^{5}+240 A^{2} B^{2}\right) x^{2} \\
-\left(640 A B^{3}+100 A^{4} B\right) x+A^{6}-32 B^{2} A^{3}-256 B^{4}
\end{array}\right.
$$

\section{THE CASE $f^{2} m$ IS DIVIDED BY 3 OR 4}

Let $s=3$ or 4 . Assume that $s \mid f^{2} m$. Let $Q=\left(x_{Q}, y_{Q}\right)$ be a point of $E[f \sqrt{-m}]$ of order s. By Propositions 2.8 and 2.9, we know $x_{Q} \in F$. We may write $y_{Q}^{2}=w^{2} \alpha_{E}$ such that $w \in F^{\times}, \alpha_{E}$ is an integer of $F$ and ideal $\left(\alpha_{E}\right)$ has no square factors. In the following, let $p$ be an odd prime number and $p$ a prime ideal of $F$ dividing $p$ and assume they satisfy the condition (1). Then there exist positive integers $u_{p}$ and $v_{p}$ such that $4 p^{\ell_{p}}=u_{p}^{2}+m f^{2} v_{p}^{2},\left(u_{p}+v_{p} f \sqrt{-m}\right) / 2 \in R,\left(u_{p}, p\right)=1$. If $u_{p}$ is even, then clearly we have $p^{\ell_{p}}=\left(u_{p} / 2\right)^{2}+m f^{2}\left(v_{p} / 2\right)^{2}$. 
THEOREM 3.1. Let $u_{p}$ and $v_{p}$ be as above. If we choose $\varepsilon_{\mathrm{p}} \in\{ \pm 1\}$ such that $\varepsilon_{\mathfrak{p}}\left(u_{p} / 2\right) \equiv\left(\alpha_{E} / \mathfrak{p}\right) \bmod s$, then we have $a_{\mathfrak{p}}(E)=\varepsilon_{\mathfrak{p}} u_{p}$.

PROOF: Since $F(Q)=F\left(\sqrt{\alpha_{E}}\right)$, we have $Q^{\sigma_{\mathfrak{p}}}=\left[\left(\alpha_{E} / \mathfrak{p}\right)\right](Q)$. Thus by Theorem 2.6, we have our assertion. It is noted that $u_{p}$ can be odd only in the case $s=3$.

Let $E_{0}$ be an elliptic curve defined by a Weierstrass equation: $y^{2}=x^{3}+A_{0} x$ $+B_{0}\left(A_{0}, B_{0} \in F\right)$. If $E_{0}$ is isomorphic to $E$ over an extension $F_{0}$ over $F$, then there exists an element $\delta \in F_{0}$ such that $A_{0}=\delta^{4} A, B_{0}=\delta^{6} B$. Since $j(E) \neq 0,1728$, we know that $A, B, A_{0}$ and $B_{0}$ are not 0 and $\delta^{2} \in F$. Therefore we may put $\alpha_{E_{0}}=\delta^{2} \alpha_{E}$. In particular we obtain

THEOREM 3.2. Let $E^{*}$ be the twist of $E$ defined by the equation $y^{2}=x^{3}+A \alpha_{E}^{2}$ $+B \alpha_{E}^{3}$. Further assume that $E^{*}$ has good reduction at $\mathfrak{p}$. Let $u_{p}$ and $v_{p}$ be as above. If we choose $\varepsilon_{\mathfrak{p}} \in\{ \pm 1\}$ such that $\varepsilon_{p}\left(u_{p} / 2\right) \equiv 1 \bmod s$, then we have $a_{\mathfrak{p}}\left(E^{*}\right)=\varepsilon_{\mathfrak{p}} u_{p}$.

The $j$-invariants of elliptic curves with complex multiplication by $R$ are solutions of the class equation $H_{\{d(R) \mid}(x)=0$ of discriminant $d(R)$ (see [3, Section 13]). In the following, we shall use the table of class equations prepared by M.Kaneko. We shall gives a canonical elliptic curve $E$ with complex multiplication by $R$ and compute $\alpha_{E}$ in subsections 3.1 and 3.2 for the cases $s=3$ and 4 respectively.

3.1. The CASE $\mathbf{s}=3$. We shall explain the process to obtain a canonical elliptic curve $E$ in the case $d(R)=-15$. At first we take a solution $j_{1}=(-191025+85995 \sqrt{5}) / 2$ of the equation:

$$
H_{15}(x)=x^{2}+191025 x-121287375=0 .
$$

Let $E_{1}$ be the elliptic curve defined by the equation: $y^{2}=x^{3}+A_{1} x+B_{1}$, where $A_{1}$ $=-1 / 48-36 /\left(j_{1}-1728\right), B_{1}=1 / 864+2 /\left(j_{1}-1728\right)$. Then the $j$-invariant of $E_{1}$ is equal to $j_{1}$. By considering twists of $E_{1}$ by elements $\sqrt{n}(n \in F=\mathbb{Q}(\sqrt{5}))$, we find an elliptic curve $E$ such that coefficients $A$ and $B$ of an equation $y^{2}=x^{3}+A x+B$ of $E$ are integers of $F$ and further the absolute value of the norm of the square factor of $A$ is as small as possible. In this case, we take $n=2^{2} 37(4+\sqrt{5}) /(\sqrt{5}(4-\sqrt{5}))$. Therefore we see $A=A_{1} n^{2}=105+48 \sqrt{5}, B=B_{1} n^{3}=-784-350 \sqrt{5}$ and

$$
\Psi_{3}(x, E)=3\left(x^{3}+6 x^{2}+3 \sqrt{5} x^{2}+(291+132 \sqrt{5}) x+590+265 \sqrt{5}\right) \times(x-6-3 \sqrt{5}) .
$$

This shows $x_{Q}=6+3 \sqrt{5}$ and $y_{Q}^{2}=2^{4}((1+\sqrt{5}) / 2)^{11}$. Finally we have

PRoposition 3.3. Let $E$ be the elliptic curve defined by the equation

$$
y^{2}=x^{3}+(105+48 \sqrt{5}) x-784-350 \sqrt{5}
$$

Then $E$ has complex multiplication by the order of discriminant -15 . Further we have $\alpha_{E}=(1+\sqrt{5}) / 2$. 
REMARK 3.4. For another root $j_{2}=(-191025-85995 \sqrt{5}) / 2$ of $H_{15}(x)=0$, we consider the conjugate elliptic curve $\bar{E}$ of $E$ over $\mathbb{Q}$ and we have $\alpha_{\bar{E}}=(1-\sqrt{5}) / 2$.

EXAMPLE 3.5 .

(1) Let $p=61$. Then $(-15 / p)=(5 / p)=1$. Thus $\ell_{\mathrm{p}}=1$. Choose the prime ideal $\mathfrak{p}$ such that $\mathfrak{p} \ni \sqrt{5}-26$. Since $\left(\alpha_{E} / \mathfrak{p}\right)=(54 / 61)=-1$ and $4 p=2^{2}+4^{2} 15, a_{\mathfrak{p}}(E)=-2$.

(2) Let $p=83$. Then $(-15 / p)=1,(5 / p)=-1$. Thus $\ell_{\mathrm{p}}=2$. Since $\left(\alpha_{E} /(p)\right)=-1$ and $4 p^{2}=154^{2}+16^{2} \cdot 15, a_{(p)}=154$.

For other cases, we give only results and data necessary to obtain the results. For each order $R$, the data consists of the class polynomial $H_{|d(R)|}(x)$, a solution $j$ of $H_{|d(R)|}=0$, coefficients $A$ and $B$ of a Weierstrass equation of an elliptic curve $E$ with $j(E)$ $=j, x_{Q}, y_{Q}^{2}$ and $\alpha_{E}$. We list them in the following format:

\begin{tabular}{|c|c|}
\hline$d(R)$ & $H_{|d(R)|}(x)$ \\
\cline { 2 - 3 } & $j$ \\
\cline { 2 - 2 } & $A, B$ \\
\cline { 2 - 2 } & $x_{Q}, y_{Q}^{2}$ \\
\cline { 2 - 2 } & $\alpha_{E}$ \\
\hline
\end{tabular}

The results and data for the case $h(R)=2$.

\begin{tabular}{|c|c|}
\hline-24 & $x^{2}-4834944 x+14670139392$ \\
\cline { 2 - 3 } & $2417472+1707264 \sqrt{2}$ \\
\cline { 2 - 3 } & $-21+12 \sqrt{2},-28+22 \sqrt{2}$ \\
\cline { 2 - 2 } & $-3+3 \sqrt{2}, 2(1-\sqrt{2})^{6}(1+\sqrt{2})$ \\
\cline { 2 - 2 }-36 & $1+\sqrt{2}$ \\
\cline { 2 - 2 } & $x^{2}-153542016 x-1790957481984$ \\
\cline { 2 - 2 } & $76771008+44330496 \sqrt{3}$ \\
\cline { 2 - 2 } & $-120-42 \sqrt{3}, 448+336 \sqrt{3}$ \\
\cline { 2 - 2 } & $3+3 \sqrt{3}, 4(2+\sqrt{3})^{2}(1+\sqrt{3})$ \\
\hline
\end{tabular}




\begin{tabular}{|c|c|}
\hline-48 & $x^{2}-2835810000 x+6549518250000$ \\
\hline & $1417905000+818626500 \sqrt{3}$ \\
\hline & $-1035-240 \sqrt{3}, 12122+5280 \sqrt{3}$ \\
\hline & $-9+18 \sqrt{3}, 4(2-\sqrt{3})^{4}(1-2 \sqrt{3})^{2}(8+6 \sqrt{3})$ \\
\hline & $8+6 \sqrt{3}$ \\
\hline-51 & $x^{2}+5541101568 x+6262062317568$ \\
\hline & $-2770550784-671956992 \sqrt{17}$ \\
\hline & $-60-12 \sqrt{17},-210-56 \sqrt{17}$ \\
\hline & $-6,-2(4-\sqrt{17})^{2}$ \\
\hline & -2 \\
\hline-60 & $x^{2}-37018076625 x+153173312762625$ \\
\hline & $(37018076625+16554983445 \sqrt{5}) / 2$ \\
\hline & $(-645+201 \sqrt{5}) / 2,1694-924 \sqrt{5}$ \\
\hline & $-(45-15 \sqrt{5}) / 2,-((1-\sqrt{5}) / 2)^{16}$ \\
\hline & -1 \\
\hline-72 & $x^{2}-377674768000 x+232381513792000000$ \\
\hline & $188837384000+77092288000 \sqrt{6}$ \\
\hline & $-470-360 \sqrt{6}, 19208+10080 \sqrt{6}$ \\
\hline & $6+9 \sqrt{6}, 4(5-2 \sqrt{6})^{2}(2+\sqrt{6})$ \\
\hline & $2+\sqrt{6}$ \\
\hline-75 & $x^{2}+654403829760 x+5209253090426880$ \\
\hline & $-327201914880+146329141248 \sqrt{5}$ \\
\hline & $-2160+408 \sqrt{5}, 42130-10472 \sqrt{5}$ \\
\hline & $\left.-(15+21 \sqrt{5}),(-25-13 \sqrt{5})(4-\sqrt{5})^{2}((1+\sqrt{5}) / 2)\right)^{14}$ \\
\hline & $-25-13 \sqrt{5}$ \\
\hline-99 & $x^{2}+37616060956672 x-56171326053810176$ \\
\hline & $-18808030478336+3274057859072 \sqrt{33}$ \\
\hline & $-45012+7836 \sqrt{33},-5198438+904932 \sqrt{33}$ \\
\hline & $-87+15 \sqrt{33},-2$ \\
\hline & -2 \\
\hline-123 & $x^{2}+1354146840576 \cdot 10^{3} x+148809594175488 \cdot 10^{6}$ \\
\hline & $-677073420288000+105741103104000 \sqrt{41}$ \\
\hline & $-960+120 \sqrt{41},-13314+2240 \sqrt{41}$ \\
\hline & $-24,-2(32+5 \sqrt{41})^{2}$ \\
\hline & -2 \\
\hline
\end{tabular}




\begin{tabular}{|c|c|}
\hline-147 & $x^{2}+34848505552896 \cdot 10^{3} x+11356800389480448 \cdot 10^{6}$ \\
\cline { 2 - 3 } & $-17424252776448000+3802283679744000 \sqrt{21}$ \\
\cline { 2 - 3 } & $-2520-240 \sqrt{21},-31724-11418 \sqrt{21}$ \\
\cline { 2 - 3 } & $63+9 \sqrt{21},(7-\sqrt{21})((5+\sqrt{21}) / 2)^{8}$ \\
\hline \multirow{4}{*}{267} & $7-\sqrt{21}$ \\
\cline { 2 - 2 } & $x^{2}+19683091854079488 \cdot 10^{6} x+$ \\
& $+531429662672621376897024 \cdot 10^{6}$ \\
\cline { 2 - 2 } & $-9841545927039744000000+1043201781864732672000 \sqrt{89}$ \\
\cline { 2 - 2 } & $-37500+3180 \sqrt{89}, 3250002-371000 \sqrt{89}$ \\
\cline { 2 - 2 } & $150,2(500+53 \sqrt{89})^{2}$ \\
\hline
\end{tabular}

The results and data for the case $h(R)=3$.

\begin{tabular}{|c|c|}
\hline-108 & $x^{3}-151013228706 \cdot 10^{3} x^{2}+224179462188 \cdot 10^{6} x$ \\
& $-1879994705688 \cdot 10^{9}$ \\
\cline { 2 - 3 } & $31710790944000 \sqrt[3]{4}+39953093016000 \sqrt[3]{2}+50337742902000$ \\
\cline { 2 - 3 } & $105 \sqrt[3]{4}-90 \sqrt[3]{2}-135,-738 \sqrt[3]{4}+738 \sqrt[3]{2}+526$ \\
\cline { 2 - 3 }-243 & $9-3 \sqrt[3]{2}, 4(1-\sqrt[3]{2})^{8}(-1+\sqrt[3]{2})$ \\
\cline { 2 - 2 } & $-1+\sqrt[3]{2}$ \\
\cline { 2 - 2 } & $-618587635244888064000-428904711070941184000 \sqrt[3]{3}$ \\
& $-297385917043138560000 \sqrt[3]{9}$ \\
\cline { 2 - 2 } & $+1560+720 \sqrt[3]{9}, 32258-11124 \sqrt[3]{3}-7704 \sqrt[3]{9}$ \\
\cline { 2 - 2 } & $42-18 \sqrt[3]{9},(-2+\sqrt[3]{9})^{8}(-4+2 \sqrt[3]{9})$ \\
\hline
\end{tabular}

3.2. The CASE $\mathbf{s}=4$. In this case, by Lemma 2.5 and Proposition 2.9, we know that $x_{Q}$ is one of two $F$-rational solutions of $\Psi_{4}(x, E) / y=0$ satisfying the condition given in the last part of Lemma 2.5. We shall explain the case $d(R)=-32$. We take a solution $j=26125000+18473000 \sqrt{2}$ of $H_{32}(x)=x^{2}-52250000 x+12167000000=0$ and consider an elliptic curve $E$ with $j(E)=j$, defined by an equation:

$$
y^{2}=x^{3}+A x+B \quad(A=-105-90 \sqrt{2}, B=630+518 \sqrt{2}) .
$$

Then $\Psi_{4}(x, E) / y=0$ has two $F$-rational solutions $x_{1}=3+5 \sqrt{2}, x_{2}=9-\sqrt{2}$. Consider a prime number $p=17=3^{2}+2^{2} 2$ and a prime ideal $\mathfrak{p}=(1-3 \sqrt{2})$. Then by counting the number of points of $\tilde{E}_{\mathfrak{p}}\left(\mathbb{F}_{p}\right)$, we know $a_{\mathfrak{p}}(E)=-6$. Since $\left(x_{1}^{3}+A x_{1}+B / \mathfrak{p}\right)$ $=(-3+3 \sqrt{2} / \mathfrak{p})=(-2 / 17)=1=(-1)^{\left(a_{p}(E) / 2-1\right)}$, we see $x_{Q}=x_{1}$. Calculating $y_{Q}^{2}$, we may obtain $\alpha_{E}=-3+3 \sqrt{2}$. For the cases $d(R)=-64,-112$, we know the class 
polynomials are:

$$
\left\{\begin{array}{l}
H_{64}(x)=x^{2}-82226316240 x-7367066619912 \\
H_{112}(x)=x^{2}-274917323970000 x+1337635747140890625
\end{array}\right.
$$

In these cases by similar argument we have $\left(E, \alpha_{E}\right)$. We list our results in the next proposition.

Proposition 3.6 .

\begin{tabular}{|l|l|l|l|}
\hline$d(R)$ & $j(E)$ & $A$ & $x_{Q}$ \\
& & $B$ & $\alpha_{E}$ \\
\hline-32 & $26125000+18473000 \sqrt{2}$ & $-105-90 \sqrt{2}$ & $9-\sqrt{2}$ \\
& & $630+518 \sqrt{2}$ & $-3+3 \sqrt{2}$ \\
\hline-64 & $41113158120+29071392966 \sqrt{2}$ & $-91-60 \sqrt{2}$ & $5+2 \sqrt{2}$ \\
& & $462+308 \sqrt{2}$ & $\sqrt{2}-1$ \\
\hline-112 & 137458661985000 & $-725-240 \sqrt{7}$ & $24-\sqrt{7}$ \\
& $+51954490735875 \sqrt{7}$ & $9520+3698 \sqrt{7}$ & 1 \\
\hline
\end{tabular}

\section{THE CASE $m f^{2}$ IS DIVIDED BY 5}

We shall consider the orders $R$ of discriminant $d(R)=-20,-35,-40,-100,-115$, -235. These orders $R$ are of class number 2. Further for any $R$, we know $F=\mathbb{Q}(\sqrt{5})$. For a given order $R$, we consider an elliptic curve $E$, defined over $F$, with complex multiplication by $R$. Proposition 2.8 shows that $\Psi_{5}(x, E)$ has only one $F$-rational factor $H_{1, E}(x)$ of degree 2 and for any solution $x_{1}$ of $H_{1, E}(x)=0$, a point $Q$ of $E$ with $x_{Q}=x_{1}$ is a generator of the group $C_{5}$. Let $L=F\left(x_{Q}\right)$ and $M=F(Q)$. For a prime number $p$ satisfying $p^{\ell_{\mathrm{p}}} \equiv 1 \bmod 5$, our problem is rather easy (see Proposition 2.7). For a prime number $p$ satisfying $p^{\ell_{p}} \equiv 4 \bmod 5$ and a prime ideal $\mathfrak{p}$ dividing $p$, to determine $r_{\mathfrak{p}}$, we must study the ideal class groups of $F$ corresponding to the fields $L$ and $M$. Regarding conductors of $L$ and $M$, we have a following result. In Proposition 4.1, we shall use the notation in Section 2.

Proposition 4.1. Let $q$ be a prime ideal of $F$ prime to $(2 \sqrt{5})$. Then $e_{\mathfrak{q}}\left(f_{L}\right)$ $=e_{\mathfrak{q}}\left(\mathfrak{f}_{M}\right)$ and $e_{\mathfrak{q}}\left(\mathfrak{f}_{M}\right) \leqslant 1$. Further if $E$ has good reduction at $\mathfrak{q}$, then $e_{\mathfrak{q}}\left(\mathfrak{f}_{M}\right)=0$.

Proof: Since $M$ is a cyclic extension of degree 4 over $F$, we have $e_{q}\left(f_{M}\right) \leqslant 1$ (see [9, Chapters IV and VI]). The other assertion is deduced from Lemma 2.10.

As for the prime ideal $(\sqrt{5})$, we have $e_{(\sqrt{5})}\left(f_{L}\right) \leqslant e_{(\sqrt{5})}\left(\mathfrak{f}_{M}\right) \leqslant 1$. Proposition 4.1 shows, to avoid tedious computation in determining class groups, it is necessary to choose an elliptic curve $E$ so that the number of prime factors of its discriminant is as small as 
possible. We shall explain the case $d(R)=-235$, because the other cases can be deduced from similar but much easier argument. First we take a solution

$$
j=-411588709724712960000-184068066743177379840 \sqrt{5}
$$

of the equation:

$$
H_{235}(x)=x^{2}+823177419449425920000 x+11946621170462723407872000=0 .
$$

We consider an elliptic curve $E$ defined by an equation: $y^{2}=x^{3}+(-15510+2068 \sqrt{5}) x$ $+(3200841-649446 \sqrt{5}) / 4$. The discriminant of $E$ is $47^{3} 2^{-4} e^{-42}, j(E)=j$ and

$$
H_{1, E}(x)=10 x^{2}+(3525-2115 \sqrt{5}) x+624160-262918 \sqrt{5}
$$

where $e=(1+\sqrt{5}) / 2$. By solving the equation $H_{1, E}(x)=0$, we obtain a generator $Q$ of $C_{5}$ given by

$$
Q=\left(3 e^{-1} t+47 e^{-10} / 2,\left(2 \sqrt{5} e^{10}\right)^{-1} \pi\right),
$$

where $t=\sqrt{47 \sqrt{5} e^{-1}}$ and $\pi=\sqrt{47 e^{-1}(2115-(211+23 \sqrt{5}) t)}$. In particular we have $L=F(t)$ and $M=L(\pi)$. Next we shall determine conductors and ideal class groups of $L$ and $M$. Since the maximal order of $L$ has a basis $\left\{1,\left(1+e^{-1} t\right) / 2\right\}$ over the maximal order of $F$, the discriminant of $L$ over $F$ is $\left(e^{-1} t\right)^{2}$. This shows that $f_{L}=(47 \sqrt{5})$. Since $M$ is real and has an imaginary conjugate field over $\mathbb{Q}$, Proposition 4.1 shows $\mathfrak{f}_{M}=\left(2^{k} \cdot 47 \sqrt{5}\right) \infty_{2}$ for some integer $k(0 \leqslant k \leqslant 2)$, where $\infty_{2}$ is the infinite place of $F$ corresponding to the conjugate embedding of $F$ to $\overline{\mathbb{Q}}$. We have only to determine the 2-exponent $k$. See [4, Section 3] for a method to calculate the 2-exponent of conductors. For a moment, we assume $M$ is defined modulo $(4 \cdot 47 \sqrt{5}) \infty_{2}$. Let $\mathfrak{P}$ be the ray class group of $F$ modulo $(4 \cdot 47 \sqrt{5}) \infty_{2}$. Denote by $\mathfrak{P}_{L}$ and $\mathfrak{P}_{M}$ the subgroups of $\mathfrak{P}$ corresponding to $L$ and $M$ respectively. Consider the ideal classes $\mathfrak{g}, \mathfrak{k}$ and $\mathfrak{l}$ of $\mathfrak{P}$ represented by the principal ideals $((1+3 \sqrt{5}) / 2),(46+47 \sqrt{5})$ and (471) respectively. Then $\mathfrak{g}$ is of order 276 and both $\mathfrak{k}$ and $I$ are of order 2 and further

$$
\mathfrak{P}=\langle\mathfrak{g}\rangle \times\langle\mathfrak{k}\rangle \times\langle\mathfrak{l}\rangle \quad \text { (a direct product). }
$$

Let $\mathfrak{P}_{1}$ be the ray class group modulo $(47 \sqrt{5})$ and $\theta$ the canonical morphism of $\mathfrak{P}$ to $\mathfrak{P}_{1}$. Then $\mathfrak{P}_{1}$ is a cyclic group generated by $\theta(\mathfrak{g})$ of order 138 and $\operatorname{Ker}(\theta)=\left\langle\mathfrak{g}^{138}, \mathfrak{k}, \mathfrak{l}\right\rangle$. Since $f_{L}=(47 \sqrt{5}), \mathfrak{P}_{L} \supset \operatorname{Ker}(\theta)$. This shows that $\mathfrak{P}_{L}=\left\langle\mathfrak{g}^{2}, \mathfrak{k}, \mathfrak{l}\right\rangle$. Next we shall determine $\mathfrak{P}_{M}$. Let $\xi$ be the endomorphism of $\mathfrak{P}$ defined by $\xi(\mathfrak{a})=\mathfrak{a}^{69}$. Then $\xi$ induces an isomorphism of $\mathfrak{P} / \mathfrak{P}_{M}$ to $\xi(\mathfrak{P}) / \xi\left(\mathfrak{P}_{M}\right)$. Consider the prime numbers $q_{1}=251=4^{2}+235, q_{2}=431$ $=14^{2}+235$ and $q_{3}=239=2^{2}+235$ and prime ideals $q_{1}=(16+\sqrt{5}), q_{2}=((43+5 \sqrt{5}) / 2)$ and $q_{3}=((31+\sqrt{5}) / 2)$ of $F$ dividing $q_{1}, q_{2}$ and $q_{3}$ respectively. In the following, for a prime ideal $\mathfrak{q}$ of $F$, we denote by $C(\mathfrak{q})$ the class of $\mathfrak{P}$ represented by $\xi(\mathfrak{q})$. Then we know $C\left(\mathfrak{q}_{1}\right), C\left(\mathfrak{q}_{2}\right)$ and $C\left(\mathfrak{q}_{3}\right)$ belong to $\mathfrak{k}, \mathfrak{k}$ and $\xi(\mathfrak{g}) \mathfrak{k}$ respectively. By counting 
the number of rational points of the reduced elliptic curve of $E$ modulo $\mathfrak{q}_{i}$, we have $a_{\mathrm{q}_{1}}(E)=-8, a_{\mathrm{q}_{2}}(E)=-28$ and $a_{\mathrm{q}_{3}}(E)=-4$. Therefore, by Theorem 2.6, we know $\mathfrak{k}, \mathfrak{l} \in \mathfrak{P}_{M}$ and the class $\xi(\mathfrak{g}) \mathfrak{k}$ corresponds to the isomorphism $\lambda$ such that $Q^{\lambda}=[3](Q)$. Since $\mathfrak{P}_{M}$ is a subgroup of $\mathfrak{P}_{L}$ of index 2 , we conclude that $\mathfrak{P}_{M}=\left\langle\mathfrak{g}^{4}, \mathfrak{k}, \mathfrak{l}\right\rangle$. In particular, $\mathfrak{P}_{M}$ does not contain the kernel $\left\langle\mathfrak{g}^{138} \mathfrak{k}, \mathfrak{l}\right\rangle$ of the canonical morphism of $\mathfrak{P}$ to the ray class group modulo $(2 \cdot 47 \sqrt{5}) \infty_{2}$. Therefore $\mathfrak{f}_{M}=(4 \cdot 47 \sqrt{5}) \infty_{2}$. Since the class $\mathfrak{m}=\xi(\mathfrak{g})$ is represented by the ideal $(743+756 \sqrt{5})$, we have

THEOREM 4.2. Let $\mathfrak{k}, \mathfrak{l}$ and $\mathfrak{m}$ be the classes of $\mathfrak{P}$ represented by the ideals $(46+47 \sqrt{5}),(471)$ and $(743+756 \sqrt{5})$ respectively. Put $\mathfrak{S}=\langle\mathfrak{m}, \mathfrak{k}, \mathfrak{l}\rangle$ and $\mathfrak{D}=\langle\mathfrak{k}, \mathfrak{l}\rangle$. Let $p$ be an odd prime number and $\mathfrak{p}$ a prime ideal of $F$ dividing $p$ and assume that they satisfy (1). Furthermore, let $u_{p}$ and $v_{p}$ be the positive integers such that $4 p^{\ell_{\mathfrak{p}}}=u_{p}^{2}+235 v_{p}^{2}$ and $\left(u_{p}, p\right)=1$. If the class $C(\mathfrak{p})$ of $\mathfrak{p}^{69}$ belongs to $\mathfrak{m}^{i} \mathfrak{D}(0 \leqslant i \leqslant 3)$, and $\varepsilon_{\mathfrak{p}} \in\{ \pm 1\}$ is chosen such that $\varepsilon_{\mathfrak{p}} u_{p} \equiv 2 \cdot 3^{i} \bmod 5$, then we have $a_{\mathfrak{p}}(E)=\varepsilon_{\mathrm{p}} u_{p}$.

REMARK 4.3. $C(\mathfrak{p}) \in \mathfrak{D} \cup \mathrm{m}^{2} \mathfrak{D}$ if and only if $p^{\ell_{\mathfrak{p}}} \equiv 1 \bmod 5$.

EXAMPLE 4.4.

(i) Let $p=239=2^{2}+235$ and $\mathfrak{p}=((31+\sqrt{5}) / 2)$. Then $C(\mathfrak{p})=\mathfrak{m} \mathfrak{k} \in \mathfrak{m} D$ and $a_{\mathfrak{p}}(E)=-4$.

(ii) Let $p=241=\left(27^{2}+235\right) / 4$ and $\mathfrak{p}=((33+5 \sqrt{5}) / 2)$. Then $C(\mathfrak{p})=\mathfrak{l} \in D$ and $a_{\mathrm{p}}(E)=27$.

(iii) Let $p=719=22^{2}+235$ and $\mathfrak{p}=((59+11 \sqrt{5}) / 2)$. Then $C(\mathfrak{p})=\mathfrak{m}^{3} \mathfrak{k l}$ $\in \mathfrak{m}^{3} D$ and $a_{\mathfrak{p}}(E)=44$.

We shall give the data and results for other cases. In the below, put $t_{m}=\sqrt{m /(\sqrt{5} e)}$ and denote by $\mathfrak{P}$ the ray class group of conductor $\mathfrak{f}_{M}$ of $F$. Further, we denote by $p$ and $\mathfrak{p}$ an odd prime number and a prime ideal of $F$ dividing $p$ such that they satisfy the condition (1) for the given elliptic curve $E$.

(I) The case $m=5, d(R)=-20$

$$
\left\{\begin{array}{l}
H_{20}(x)=x^{2}-1264000 x-681472000 \\
j(E)=632000+282880 \sqrt{5} \\
A=-50 / 3-5 \sqrt{5}, B=100 / 3+280 \sqrt{5} / 27 \\
x_{Q}=5 e^{2} / 6+t_{m}, y_{Q}=(\sqrt{5})\left(e+t_{m}\right) \sqrt{1+t_{m}^{-1}} \\
L=F\left(t_{m}\right), M=L\left(\sqrt{1+t_{m}^{-1}}\right), \mathfrak{f}_{L}=(4 \sqrt{5}), \mathfrak{f}_{M}=(8 \sqrt{5}) \\
\mathfrak{P}=\left\langle\mathfrak{g}_{1}\right\rangle \times\left\langle\mathfrak{g}_{2}\right\rangle, \mathfrak{P}_{L}=\left\langle\mathfrak{g}_{1}^{2}, \mathfrak{g}_{2}\right\rangle, \mathfrak{P}_{M}=\left\langle\mathfrak{g}_{1}^{2} \mathfrak{g}_{2}\right\rangle
\end{array}\right.
$$

where $g_{1}$ and $g_{2}$ are the classes of order of 4 and 2 represented by the ideals $((21+\sqrt{5}) / 2)$ and $(11+2 \sqrt{5})$.

PROPOSITION 4.5. Let $u_{p}$ and $v_{p}$ be the positive integers such that $p^{\ell_{p}}$ 
$=u_{p}^{2}+5 v_{p}^{2},\left(u_{p}, p\right)=1$. Choose $\varepsilon_{\mathfrak{p}} \in\{ \pm 1\}$ such that $\varepsilon_{\mathfrak{p}} u_{p} \equiv 2^{i} \bmod 5$ if the class of $\mathfrak{p}$ belongs to $\mathfrak{g}_{1}^{i} \mathfrak{P}_{M}(0 \leqslant i \leqslant 3)$. Then we have $a_{\mathfrak{p}}(E)=2 \varepsilon_{\mathfrak{p}} u_{p}$.

Choosing a suitable generator of $\mathfrak{p}, p$ is written in a form $p=a^{2}-5 b^{2}$, where $a$ and $b$ are integers satisfying the condition:

$$
\begin{aligned}
& a \equiv\left\{\begin{array}{lll}
1 & \bmod 20 & \text { if } p \equiv 1 \bmod 5 \\
17 & \bmod 20 & \text { if } p \equiv 4 \bmod 5,
\end{array}\right. \\
& b \equiv\left\{\begin{array}{llll}
0 & \bmod 4 & \text { if } p \equiv 1 \bmod 8 \\
2 & \bmod 4 & \text { if } p \equiv 5 \bmod 8
\end{array}\right.
\end{aligned}
$$

For $i=1,2$, let $p_{i}=a_{i}^{2}-5 b_{i}^{2}$ be the prime numbers represented as above. If $p_{1} \equiv p_{2}$ mod 40, then prime ideals $\left(a_{1}+b_{1} \sqrt{5}\right)$ and $\left(a_{2}+b_{2} \sqrt{5}\right)$ belong to the same class of $\mathfrak{P}$ if and only if $a_{1}-a_{2}+5\left(b_{1}-b_{2}\right) \equiv 0 \bmod 40$. Let $\mathfrak{T}=\left\langle\mathfrak{g}_{1}^{2}\right\rangle$. Then we see if $p \equiv 1$ (respectively $9,21,29) \bmod 40$, then the class $C(\mathfrak{p})$ of the prime ideal $\mathfrak{p}=(a+b \sqrt{5})$ belongs to $\mathfrak{T}$, (respectively $\mathfrak{g}_{2} \mathfrak{g}_{1} \mathfrak{T}, \mathfrak{g}_{2} \mathfrak{T}, \mathfrak{g}_{1} \mathfrak{T}$ ) and furthermore $C(\mathfrak{p}) \in \mathfrak{P}_{M}$ if and only if $a+5 b \equiv 1$ (respectively $-3,11,7) \bmod 40$. Therefore we have

PROPOSITION 4.6. Let $p=u^{2}+5 v^{2}=a^{2}-5 b^{2}$, where $u$ and $v$ are positive integers and $a$ and $b$ are integers satisfying the above condition. Then if we choose $\varepsilon_{\mathfrak{p}} \in\{ \pm 1\}$ such that

$$
\varepsilon_{\mathfrak{p}} u \equiv\left\{\begin{array}{lll}
(-1)^{(a+5 b-1) / 20} a \bmod 5 & \text { if } p \equiv 1 \bmod 40 \\
(-1)^{(a+5 b+3) / 20} a \bmod 5 & \text { if } p \equiv 9 \bmod 40 \\
(-1)^{(a+5 b-11) / 20} a \bmod 5 & \text { if } p \equiv 21 \bmod 40 \\
(-1)^{(a+5 b-7) / 20} a & \bmod 5 & \text { if } p \equiv 29 \bmod 40
\end{array}\right.
$$

then we have $a_{\mathfrak{p}}(E)=2 \varepsilon_{\mathfrak{p}} u$.

(II) The case $m=35, d(R)=-35$

$$
\left\{\begin{array}{l}
H_{35}(x)=x^{2}+117964800 x-134217728000 \\
j(E)=-58982400-26378240 \sqrt{5} \\
A=-70 \sqrt{5} / 3, B=(13475+980 \sqrt{5}) / 108 \\
x_{Q}=\left(35 e-3 t_{m}\right) / 6 e^{2}, y_{Q}=\left(t_{m}^{2} / 2 e^{2}\right) \sqrt{\sqrt{5}-(9+\sqrt{5})\left(e t_{m}\right)^{-1}} \\
L=F\left(t_{m}\right), M=L\left(\sqrt{\sqrt{5}-(9+\sqrt{5})\left(e t_{m}\right)^{-1}}\right) \\
\mathfrak{f}_{L}=(7 \sqrt{5}), \mathfrak{f}_{M}=\left(14 \sqrt{5} \infty_{2}\right), \\
\mathfrak{P}=\langle\mathfrak{h}\rangle, \mathfrak{P}_{L}=\left\langle\mathfrak{h}^{2}\right\rangle, \mathfrak{P}_{M}=\left\langle\mathfrak{h}^{4}\right\rangle,
\end{array}\right.
$$

where $\mathfrak{h}$ is the class of order 12 represented by the ideal $(6+\sqrt{5})$. 
Proposition 4.7. Let $u_{p}$ and $v_{p}$ be the positive integers such that $4 p^{\ell_{p}}$ $=u_{p}^{2}+35 v_{p}^{2},\left(u_{p}, p\right)=1$. Choose $\varepsilon_{\mathfrak{p}} \in\{ \pm 1\}$ such that $\varepsilon_{p} u_{p} \equiv 2 \cdot 3^{i} \bmod 5$ if the class of $\mathfrak{p}$ belongs to $\mathfrak{h}^{i} \mathfrak{P}_{M}(0 \leqslant i \leqslant 3)$. Then we have $a_{\mathfrak{p}}(E)=\varepsilon_{\mathfrak{p}} u_{p}$.

(III) The case $m=10, d(R)=-40$

$$
\left\{\begin{array}{l}
H_{40}(x)=x^{2}-425692800 x+9103145472000 \\
j(E)=212846400+95178240 \sqrt{5}, \\
A=-125+15 \sqrt{5}, B=-200+240 \sqrt{5} \\
x_{Q}=\left(10 e+t_{m}\right) / e^{2}, y_{Q}=2 e^{-2} t_{m} \sqrt{15 e^{-1}+(40-11 \sqrt{5}) t_{m}^{-1}}, \\
L=F\left(t_{m}\right), M=L\left(\sqrt{15 e^{-1}+(40-11 \sqrt{5}) t_{m}^{-1}}\right), \\
\mathfrak{f}_{L}=(8 \sqrt{5}), \mathfrak{f}_{M}=\left(16 \sqrt{5)} \infty_{2},\right. \\
\mathfrak{P}=\langle\mathfrak{g}\rangle \times\langle\mathfrak{h}\rangle \times\langle\mathfrak{l}), \mathfrak{P}_{L}=\left\langle\mathfrak{g}^{2}, \mathfrak{h}, \mathfrak{l}\right\rangle, \mathfrak{P}_{M}=\langle\mathfrak{h}, \mathfrak{l}\rangle,
\end{array}\right.
$$

where $\mathfrak{g}, \mathfrak{h}$ and $\mathfrak{l}$ are the classes of order 4,4 and 2 represented by the ideals $(6+\sqrt{5})$, $((53+3 \sqrt{5}) / 2)$ and $((37+7 \sqrt{5}) / 2)$ respectively.

Proposition 4.8. Let $u_{p}$ and $v_{p}$ be the positive integers such that $p^{\ell_{p}}$ $=u_{p}^{2}+10 v_{p}^{2},\left(u_{p}, p\right)=1$. Choose $\varepsilon_{p} \in\{ \pm 1\}$ such that $\varepsilon_{p} u_{p} \equiv 2^{i} \bmod 5$ if the class of $\mathfrak{p}$ belongs to $\mathfrak{g}^{i} \mathfrak{P}_{M}(0 \leqslant i \leqslant 3)$. Then we have $a_{\mathfrak{p}}(E)=2 \varepsilon_{\mathfrak{p}} u_{p}$.

(IV) The case $m=1, d(R)=-100$

$$
\left\{\begin{array}{l}
H_{100}(x)=x^{2}-44031499226496 x-292143758886942437376 \\
j(E)=22015749613248+9845745509376 \sqrt{5} \\
A=-3000-805 \sqrt{5}, B=56000+32200 \sqrt{5} \\
x_{Q}=e^{-2} \sqrt{5}\left((\sqrt{5} e)^{3}+t_{m}\right) \\
y_{Q}=e^{-2} \sqrt{5} \sqrt{30 \sqrt{5}+(103+11 \sqrt{5}) t_{m}} \\
L=F\left(t_{m}\right), M=L\left(\sqrt{30 \sqrt{5}+(103+11 \sqrt{5}) t_{m}}\right) \\
\mathfrak{f}_{L}=(4 \sqrt{5}), \mathfrak{f}_{M}=(8 \sqrt{5}) \infty_{2} \\
\mathfrak{P}=\left\langle\mathfrak{k}_{1}\right\rangle \times\left\langle\mathfrak{k}_{2}\right\rangle \times\left\langle\mathfrak{k}_{3}\right\rangle, \mathfrak{P}_{L}=\left\langle\mathfrak{k}_{1}^{2}, \mathfrak{k}_{2}, \mathfrak{k}_{3}\right\rangle \\
\mathfrak{P}_{M}=\left\langle\mathfrak{k}_{2}, \mathfrak{k}_{3}\right\rangle
\end{array}\right.
$$

where $\mathfrak{k}_{1}, \mathfrak{k}_{2}$ and $\mathfrak{k}_{3}$ are the classes represented by the ideals $((21+\sqrt{5}) / 2),(11+2 \sqrt{5})$ and $(58+\sqrt{5})$ and the order of $\mathfrak{k}_{1}, \mathfrak{k}_{2}$ and $\mathfrak{k}_{3}$ are 4,2 and 2 respectively.

PROPOSITION 4.9. Let $u_{p}$ and $v_{p}$ be the positive integers such that $p^{\ell_{p}}=u_{p}^{2}+25 v_{p}^{2},\left(u_{p}, p\right)=1$. Choose $\varepsilon_{p} \in\{ \pm 1\}$ such that $\varepsilon_{p} u_{p} \equiv 2^{i} \bmod 5$ if the class of $\mathfrak{p}$ belongs to $\mathfrak{k}_{1}^{i} \mathfrak{P}_{M}(0 \leqslant i \leqslant 3)$. Then we have $a_{\mathfrak{p}}(E)=2 \varepsilon_{\mathfrak{p}} u_{p}$. 
(V) The case $m=115, d(R)=-115$

$$
\left\{\begin{array}{l}
H_{115}(x)=x^{2}+427864611225600 x+130231327260672000 \\
j(E)=-213932305612800+95673435586560 \sqrt{5} \\
A=-345-23 \sqrt{5}, B=-(19573+5290 \sqrt{5}) / 4 \\
x_{Q}=e^{3} \sqrt{5} t_{m}\left(\sqrt{5} t_{m}+e^{4}\right) / 10 \\
y_{Q}=\left(e^{9} t_{m}^{2} / 10\right) \sqrt{15 e^{-1}+(-85+61 \sqrt{5}) t_{m}^{-1}} \\
L=F\left(t_{m}\right), M=L\left(\sqrt{15 e^{-1}+(-85+61 \sqrt{5}) t_{m}^{-1}}\right) \\
\mathfrak{f}_{L}=(23 \sqrt{5}), \mathfrak{f}_{M}=(92 \sqrt{5}) \infty_{2} \\
\mathfrak{P}=\left\langle\mathfrak{f}_{1}\right\rangle \times\left\langle\mathfrak{f}_{2}\right\rangle \times\left\langle\mathfrak{f}_{3}\right\rangle, \mathfrak{P}_{L}=\left\langle\mathfrak{f}_{1}^{2}, \mathfrak{f}_{2}, \mathfrak{f}_{3}\right\rangle \\
\mathfrak{P}_{M}=\left\langle\mathfrak{f}_{1}^{4}, \mathfrak{f}_{2}, \mathfrak{f}_{3}\right\rangle
\end{array}\right.
$$

where $\mathfrak{f}_{1}, \mathfrak{f}_{2}$ and $\mathfrak{f}_{3}$ are the classes represented by the ideals $((1+3 \sqrt{5}) / 2),(24+23 \sqrt{5})$ and (91) and the order of $\mathfrak{f}_{1}, \mathfrak{f}_{2}$ and $\mathfrak{f}_{3}$ are 132,2 and 2 respectively. Since the map $\xi_{1}: \mathfrak{a} \rightarrow \mathfrak{a}^{33}$ of $\mathfrak{P}$ to itself induces an isomorphism of $\mathfrak{P} / \mathfrak{P}_{M}$ to $\xi_{\mathbf{1}}(\mathfrak{P}) / \xi_{1}\left(\mathfrak{P}_{M}\right)$ and $\mathfrak{f}_{0}=\mathfrak{f}_{1}^{33}$ is represented by the ideal $(423+372 \sqrt{5})$, we have

PROPOSITION 4.10. Let $\mathfrak{S}=\left\langle\mathfrak{f}_{0}, \mathfrak{f}_{2}, \mathfrak{f}_{3}\right\rangle$ and $\mathfrak{D}=\left\langle\mathfrak{f}_{2}, \mathfrak{f}_{3}\right\rangle$. Let $u_{p}$ and $v_{p}$ be the positive integers such that $4 p^{\ell_{p}}=u_{p}^{2}+115 v_{p}^{2},\left(u_{p}, p\right)=1$. Choose $\varepsilon_{p} \in\{ \pm 1\}$ such that $\varepsilon_{\mathfrak{p}} u_{p} \equiv 2 \cdot 3^{i} \bmod 5$ if the class of $\mathfrak{p}^{33}$ belongs to $\mathfrak{f}_{0}^{i} \mathfrak{D}(0 \leqslant i \leqslant 3)$. Then we have $a_{\mathfrak{p}}(E)=\varepsilon_{\mathfrak{p}} u_{p}$.

\section{REFERENCES}

[1] B.W. Brewer, 'On certain character sums', Trans. Amer. Math. Soc. 99 (1961), 241-245.

[2] B.W. Brewer, 'On primes of the form $u^{2}+5 v^{2}$, Proc. Amer. Math. Soc. 17 (1966), 502-509.

[3] D.A. Cox, Primes of the form $x^{2}+n y^{2}$ (John Wiley \& Sons Inc., New York, 1989).

[4] T. Hiramatsu and N. Ishii, 'Quartic residuacity and cusp forms of weight one', Comment Math. Univ. St. Paul. 34 (1985), 91-103.

[5] A. Joux and F. Morain, 'Sur les sommes de caractères liées aux courbes elliptiques à multiplication complexe', J. Number Theory 55 (1995), 108-128.

[6] S. Lang, Elliptic functions (Addison-Wesley Publishing Co. Inc., 1973).

[7] F. Leprévost and F. Morain, 'Revêtements de courbes elliptiques à multiplication complexe par des courbes hyperelliptiques et sommes de caractères', J. Number Theory 64 (1997), 165-182.

[8] H.W. Lenstra, Jr., 'Complex multiplication structure of elliptic curves', J. Number Theory 56 (1996), 227-241.

[9] J-P. Serre, Local fields, Graduate Texts in Mathematics 67 (Springer-Verlag, New York, 1979). 
[10] J.H. Silverman, The arithmetic of elliptic curves, Graduate Texts in Mathematics 106 (Springer-Verlag, New York, 1986).

[11] J.H. Silverman, Advanced topics in the arithmetic of elliptic curves, Graduate Texts in Mathematics 151 (Springer-Verlag, New York, 1994).

Department of Mathematics and Information Science Osaka Prefecture University

1-1 Gakuen-cho, Sakai

Osaka

599-8531 Japan

e-mail: ishii@mi.cias.osakafu-u.ac.jp 